\section{Nature's Allograft}

Nature's Transplant. The Transplantation Immunology of Viviparity. By J. Maxwell Anderson. Pp. viii +145. (Butterworth: London, November 1972.) £3.

A MAJOR unsolved problem in transplantation biology is how the mammalian embryo manages to survive in the uterine environment of a genetically differing female without eliciting the expected immunological rejection reactions. The embryo seems to be exempt from the "laws of transplantation immunity", and as such is of considerable interest not cnly for its own sake but for the possibility of providing valuable clues to the attainment of successful surgical grafts of tissues and organs and to an understanding of the cancer-host relationship.

The precise nature of the problem was defined in 1953 in an eloquent essay by Medawar ${ }^{1}$, and most of the experiments subsequently carried out in attempts to elucidate this paradox of transplantation immunology have been within the philosophical framework of that assay. Maxwell Anderson's short book is essentially a contemporary assessment of the old problem. It deals comprehensively with various theories put forward by Medawar and others, and considers much evidence to support or undermine them. The embryo's survival is undoubtedly due to a number of biological adaptations, including the specialized nature of its vascularization, the selective placental "barrier" restricting any largescale traffic of maternal immunologically competent cells, and the establishment of a complex foeto-maternal immunological equilibrium following exchange of antigenic information. The presence of blocking antibody is thought to be one of the factors involved in this equilibrium.

Despite the blurb's assertion that "This is the first unified account of the subject", Anderson's analysis is very much in line with many of the reviews that have appeared in the past four years, although his "Integrative Hypothesis" of chapter 7 does include one or two new thoughts. The book contains eight plates on embryology and tissue grafting in the armadillo, which might be thought excessive considering the wide scope of the text.

In general, it is difficult to see the market for this book. It is far too detailed for anyone not involved in research in the field (no fewer than seventy pages out of the total of 145 are taken up by reference lists), whilst those that are would probably find Beer and Billingham's recent review ${ }^{2}$ of more value.

W. D. Billington

${ }^{1}$ Medawar, P. B., Symp. Soc. Exp. Biol., 7, 320 (1953).

2 Beer, A. E., and Billingham, R. E., Adv. Immunoi., 14, 1 (1971).

\section{Solid State Spectroscopy}

Optical Properties of Solids. By Frederick Wooten. Pp. xiii +260 . (Academic: New York and London, October 1972.) $\$ 12.95$.

SPECTROSCOPIC studies provide a wealth of information about the various excitations which occur in crystals, and in spite of the very large amount of research which has already been carried out, solid-state spectroscopy still throws up a steady flow of new phenomena, The book under review aims to supply a distillation of this active research field, with the fundamental principles of the subject described at a level suitable for firstyear graduate students. The material is restricted to the optical properties associated with electronic transitions in perfect crystals. There is some discussion of surface effects in addition to the more familiar bulk properties of electronic excitations in the effectivelyinfinite crystal. The author is concerned throughout the book to explain the theoretical concepts behind the spectroscopic properties. He has taken pains to provide simple derivations of the main theoretical expressions, and it seems to me that his mixture of mathematical rigour and physical insight achieves a suitable balance for his intended readers. The theoretical results are illustrated throughout the book by a well-chosen selection of experimental data, but the emphasis of the work is theoretical, and there is no attempt to provide a survey of experimental results or techniques.

The main topic of the book is the interaction of electromagnetic radiation with electronic transitions. The quantum mechanics of transition rates, absorption and dispersion of radiation is presented in some detail. The dielectric function is used throughout as a bridge between the microscopic electronic properties of solids and the macroscopic concepts used in the description of optical phenomena. The associated theoretical machinery of linear response functions and the fluctuation-dissipation theorem is covered, with particular reference to electronic spectroscopy. Typical spectra of metals, alloys and semiconductors are described and interpreted in terms of the theory. The techniques of photoemission and characteristic energy-loss spectroscopy are shown to be valuable for the complementary information they can provide on the electronic energy-band structure.

The book can be recommended to readers with a good basic knowledge of solid-state physics and quantum mechanics who need a more specialized introduction to electronic spectroscopy. The style of writing and the printing are very readable, and the book is well produced except for a number of misprints which include the complete omission of equation (3.75) from the review copy.

RODNEY LOUDON

\section{Multivariate Statistics}

Multivariate Statistical Analysis: a Bibliography. By T. W. Anderson, Somesh Das Gupta and George P. H. Styan. Pp. $x+642$. (Oliver and Boyd: Edinburgh, 1972.) $£ 10$.

THIs new bibliography is one of those immeasurably important pieces of professional housekeeping to which no review can ever do full justice. Recalling that the senior author, Anderson, is the doyen of statisticians in this field of analysis, it is interesting to note that the project started (in 1963) from the desire of the three authors to understand and increase their own knowledge of the subject. This leads to a systematic search of the literature and on to a massive cooperative organization involving a computer-produced listing and large-scale photography of typescript.

The first chapter covers the available books, a classified list of 213 titles together with an author index, available in 1970 or earlier. This is followed by a chapter on the structure of the 819 journals and other collections of papers used, and here the terminal date is 1966 , although there are some items in the Addenda at the end of the volume. The third chapter contains the core bibliography of the (nearly) 6,100 papers arranged alphabetically by author with analysis by year, language, numbers of authors and papers. This, of course, is the computer-produced portion and is a fine example of its kind; the organization is of general bibliographic interest. The collection of papers is indexed in chapter 4 in accordance with a subject-matter code described in detail in chapter 5 where is also found a straightforward index to this subject-matter code.

The preface contains a clear statement of the conditions for complete or selective coverage of the various aspects of this immense field of statistical techniques. At once it illustrates how real boundaries are growing less distinct as well as the need to draw some boundaries or be selective if any usable work is to result.

The statistical profession's collection of works of this kind is steadily improving and the authors, together with everyone associated with ABOMSA (as it has been affectionately styled), merit warmest congratulations and thanks for their magnificent contribution.

W. R. BUCKLAND 